Bull. Austral. Math. Soc.

$30 \mathrm{D} 55$

VOL. $42(1990) \quad[417-425]$

\title{
LINEAR FUNCTIONALS ON SOME WEIGHTED BERGMAN SPACES
}

\section{MAHER M.H. MarzUQ}

The weighted Bergman space $A^{p_{1} \alpha}, 0<p<1, \alpha>-1$ of analytic functions on the unit disc $\triangle$ in $C$ is an $F$-space. We determine the dual of $A^{p_{1} \alpha}$ explicitly.

\section{INTRODUCTION}

Let $\triangle$ be the unit disc in $C$. For a function analytic in $\Delta$, we write

$$
\begin{aligned}
M_{p}(r, f) & =\left(\frac{1}{2 \pi} \int_{0}^{2 \pi}\left|f\left(r e^{i \theta}\right)\right|^{p} d \theta\right)^{1 / p}, 0<p<\infty \\
M_{\infty}(r, f) & =\max _{0 \leqslant \theta<2 \pi}\left|f\left(r e^{i \theta}\right)\right| .
\end{aligned}
$$

It is well known that $M_{p}(r, f)(0<p \leqslant \infty)$ is an increasing function of $r(0 \leqslant r<1)$.

The Hardy space $H^{p}(0<p \leqslant \infty)$ is the class of analytic functions $f$ in $\triangle$ and

$$
\|f\|_{p}=\sup _{0 \leqslant r<1} M_{p}(r, f)<\infty .
$$

The weighted Bergman space $A^{p, \alpha}, p>0, \alpha>-1$, is the class of analytic functions in $\triangle$ for which

$$
\|f\|_{p, \alpha}=\left(\frac{\alpha+1}{\pi} \iint_{\Delta}(1-|z|)^{\alpha}|f(z)|^{\dot{p}} d x d y\right)^{1 / p}<\infty .
$$

$A^{p, \alpha} 1 \leqslant p<\infty, \alpha>-1$ is known to be a Banach space and a Fréchet space with the metric

$$
\|f\|_{p, \alpha}^{p}=\frac{\alpha+1}{\pi} \iint_{\Delta}(1-|z|)^{\alpha}|f(z)|^{p} d x d y
$$

for $0<p<1$. Although $A^{p, \alpha}, 0<p<1, \alpha>-1$ is not locally convex, it nevertheless has enough continuous linear functionals to separate points [8]. It is clear that $A^{p, 0}=$ $A^{p}$ where $A^{p}$ is the usual Bergman space; also $H^{p} \subset A^{p(\alpha+2), \alpha}[6]$.

Received 7 December 1989

Copyright Clearance Centre, Inc. Serial-fee code: 0004-9729/90 \$A2.00+0.00. 
Duren, Romberg and Shields [2, Theorem 1] have studied linear functionals on $H^{p}$ over the unit disc $\triangle$ for $0<p<1$. Shapiro computed the dual space $A^{p, \alpha}$ $(0<p<1, \alpha>-1)$ by determining the Mackey topology of $A^{p, \alpha}[8]$. Motivated by their work we shall prove a main theorem in Section 3 which gives the explicit dual of $A^{p, \alpha}$ spaces with $0<p<1$ and $\alpha>-1$.

In Section 4 we prove a theorem which says that $\left(A^{p, \alpha}\right)^{*}$ is topologically equivalent to a certain Banach space $\Lambda_{\gamma}^{m-2}\left(\Lambda_{*}^{m-2}\right)$.

Throughout this paper $C$ denotes a positive constant, not necessarily the same at each occurrence.

\section{Preliminaries}

Let $F(z)$ be analytic in $\triangle$. Then $F(z)$ is said to belong to the Lipschitz space $\Lambda_{\beta}$ if

$$
\sup _{|t-s|<h}\left|F\left(e^{i t}\right)-F\left(e^{i s}\right)\right|=O\left(h^{\beta}\right) \quad(0 \leqslant \beta<1)
$$

A continuous function $F(z)$ is said to belong to the class $\Lambda_{*}$ if

$$
|F(t+h)-2 F(t)+F(t-h)|=O(h)
$$

uniformly in $t$. If $\beta>0$ and $f(z)=\sum_{n=0}^{\infty} a_{n} z^{n}$ is analytic in $\Delta$, the fractional derivative of order $\beta$ is

$$
f^{[\beta]}(z)=\sum_{n=0}^{\infty} \frac{\Gamma(n+1+\beta)}{n !} a_{n} z^{n},
$$

the fractional integral of order $\beta$ is

$$
f_{[\beta]}(z)=\sum_{n=0}^{\infty} \frac{n !}{\Gamma(n+1+\beta)} a_{n} z^{n}
$$

and $f^{[\beta]}, f_{[\beta]}$ are analytic in $\Delta[2]$.

Let $A(\Delta)$ denote the class of analytic functions in $\Delta$ and continuous on $\bar{\Delta}$. For analytic functions $f(z)$ we write $f \in \Lambda_{\beta}\left(\Lambda_{*}\right)$ to indicate that $f \in A$ and the boundary $f\left(e^{i \theta}\right)$ is in $\Lambda_{\beta}\left(\Lambda_{*}\right)$.

We need the following theorems:

Theorem A. [2]. Let $f$ be analytic in $\triangle$. Then $f \in \Lambda_{\beta}(0<\beta \leqslant 1)$ if and only if

$$
f^{\prime}(z)=O\left(\frac{1}{(1-r)^{1-\beta}}\right) .
$$


THEOREM B. [2]. Let $f$ be analytic in $\Delta$. Then $f \in \Lambda_{*}$ if and only if

$$
f^{\prime \prime}(z)=O\left(\frac{1}{1-r}\right) \text {. }
$$

Theorem C. Let $f \in A^{p, \alpha}, 0<p<q<\infty$. Then

$$
\int_{0}^{1}(1-\rho)^{(\alpha+2) q / p-2} M_{q}^{q}(\rho, f) d \rho<\infty .
$$

The proof is a consequence of the following inequality

$$
|f(z)| \leqslant \frac{C\|f\|_{p, \alpha}}{\left(1-|z|^{2}\right)^{(\alpha+2) / p}}
$$

which holds for $f \in A^{p, \propto}[8]$.

By using Theorem $\mathrm{C}$ and (2.1) we have the following theorem:

TheOREM D. Let $f \in A^{p, \alpha}, 0<p<q<\infty$. Then

$$
\int_{0}^{1}(1-\rho)^{(\alpha+2) q / p-3} J(\rho, f) d \rho<\infty,
$$

where $J(\rho, f)=\int_{0}^{\rho} M_{q}^{q}(r, f) d r$.

THEOREM E. [4]. Let $f$ be analytic in $\Delta$ and $0<q \leqslant 1$. Then

$$
\lim _{\rho \rightarrow 1} J\left(s, f_{[\beta]}\right) \leqslant C \int_{0}^{1}(1-\rho)^{q \beta-1} J(\rho, f) d \rho .
$$

Theorem F. Let $f \in A^{p, \alpha}, 0<p<q \leqslant 1$ and $0<\beta<(\alpha+2) / p$. Then $f_{[\beta]} \in A^{q}$ where $q=2 p /((\alpha+2)-\beta p)$.

The proof follows from Theorems $E$ and $D$.

THEOREM G. [2]. If $f$ is analytic in $\Delta$ and $f^{\prime}(z)=O(1 /(1-r))$, then

$$
f^{[1 / 2]}(z)=O\left(\frac{1}{(1-r)^{1 / 2}}\right) .
$$

TheOREM H. [9]. Suppose $\alpha>-1$ and $\gamma>1+\alpha$; then for $0<r, \rho<1$,

$$
\int_{0}^{1} \frac{(1-r)^{\alpha}}{(1-\rho r)^{\gamma}} d r=O\left(\frac{1}{1-\rho}\right)^{\alpha-\gamma+1} .
$$

THEOREM I. [10, p.128]. Let $f(z)=\sum_{k=0}^{\infty} a_{n} z^{n} \in A^{p}, 0<p \leqslant 1$ and $\alpha>-1$. Then $\left|a_{n}\right| \leqslant C n^{[(\alpha+2) / p]-1}$. 


\section{REPRESENTATION OF BOUNDED LINEAR FUNCTIONALS}

Let $T$ be a linear bounded functional on $A^{p, \alpha}(0<p<1, \alpha>-1)$. Then $T \in$ $\left(A^{p, \alpha}\right)^{*}$ if and only if

$$
\|T\|=\sup _{\|f\|_{p, \alpha<1}}|T(f)|<\infty .
$$

It follows that

$$
|T(f)| \leqslant\|T\|\|f\|_{p, \alpha}
$$

for all $f \in A^{p, \alpha}$. Here $\left(A^{p, \alpha}\right)^{*}$ is a Banach space.

Theorem 1 gives a representation for bounded linear functionals $T$ on $A^{p, \alpha}$ $(0<p<1, \alpha>-1)$.

THEOREM 1. Let $T \in\left(A^{p, \alpha}\right)^{*}, 0<p<1$. Then there is a unique function $g \in A$ such that

$$
T(f)=\frac{1}{2 \pi} \iint f(z) g(\bar{z}) d x d y .
$$

If $(\alpha+2) /(m+1)<p<(\alpha+2) / m, m=2,3, \ldots$, then

$$
g^{(m-2)} \in \Lambda_{(\alpha+2) / p-m}
$$

Conversely, for any $g$ with $g^{(m-2)} \in \Lambda_{(\alpha+2) / p-m}$ the double integral (3.1) exists for all $f \in A^{p, \alpha}$ and defines a functional $T \in\left(A^{p, \alpha}\right)^{*}$.

If $p=(\alpha+2) /(n+1)$, then $g^{(m-2)} \in \Lambda_{*}$.

Conversely, for any $g$ with $g^{(m-2)} \in \Lambda_{*}$, the double integral (3.1) exists and represents a bounded linear functional on $A^{p, \alpha}$.

Theorem 1 of [2] for $0<p<1 / 2$ can be regarded as the limiting case of $\alpha=-1$ of our results and the question arises whether it holds for the case $1 / 2<p<1$. Also, this result generalises the announced result of Burchaev and Ryabykh [1] for $A^{p}$.

Proof: Suppose that $T \in\left(A^{p, \alpha}\right)^{*}$ and $T z^{k}=b_{k} /(2(k+1))$; then $\left|T z^{k}\right| \leqslant$ $\|T\|\left\|z^{k}\right\|_{p, \alpha}$. But

$$
\left\|z^{k}\right\|_{p, \alpha}=\left(\frac{\alpha+1}{\pi} \int_{0}^{1} \int_{0}^{2 \pi}(1-r)^{\alpha} r^{p k+1} d r d \theta\right)^{1 / p} \leqslant C k^{-(1+\alpha) / p}
$$

[5], so $\left|b_{k}\right| \leqslant C\|T\| / k^{(1+\alpha) / p-1}$ and hence $g(z)=\sum_{k=0}^{\infty} b_{k} z^{k}$ is analytic in $\triangle$. For each $f(z)=\sum_{k=0}^{\infty} a_{k} z^{k} \in A^{p, \alpha}$ and for fixed $\rho \in[0,1)$ let $f_{\rho}(z)=f\left(\rho^{2} z\right)$. Because the power series of $f_{\rho}$ converges uniformly on $\Delta$, and because $T$ is continuous, we have

$$
T\left(f_{\rho}(z)\right)=\sum_{k=0}^{\infty} a_{k} \frac{b_{k}}{2(k+1)} \rho^{2 k} .
$$


As $\rho \rightarrow 1, f_{\rho} \rightarrow f$ in the $A^{p, \alpha}$ metric $[8$, p.197]

$$
\begin{aligned}
T(f) & =\lim _{\rho \rightarrow 1} \frac{1}{2 \pi} \iint_{|z|<p} \sum_{k=0}^{\infty} a_{k} z^{k} \sum_{k=0}^{\infty} b_{k} \bar{z}^{k} d x d y \\
& =\frac{1}{2 \pi} \iint_{|z|<1} f(z) g(\bar{z}) d x d y .
\end{aligned}
$$

For fixed $\rho \in \Delta$, let $f_{\xi}(z)=2 /(1-\xi z)^{2}=\sum_{n=0}^{\infty}(2 n+2) z^{n} \xi^{n}$. Then

$$
|g(\xi)|=|T(f)| \leqslant\|T\|\left\|\frac{2}{(1-\xi z)^{2}}\right\|_{p, \alpha}
$$

and hence $g \in H^{\infty}(\Delta)$; also $g \in A$, since $\lim _{\xi \rightarrow 1} g(\xi)=\lim _{\xi \rightarrow 1} T(f(z))$, and hence $\lim _{\xi \rightarrow 1} g(\xi)=T\left(f_{1}\right)$.

$$
\begin{aligned}
& \text { If }(\alpha+2) /(m+1)<p<(\alpha+2) / m, m=2,3, \ldots, \text { let } \\
& \qquad F(z)=\frac{d^{m}}{d \xi^{m}}\left(\frac{\xi}{1-\xi z}\right), \quad|\xi|<1 .
\end{aligned}
$$

By a calculation, since $F \in A^{p, \alpha}$ we get

$$
T(F)=\frac{1}{2} g^{(m-1)}(\xi) .
$$

It now follows from Theorem $B$ that

$$
\left|g^{m-1}(\xi)\right| \leqslant 2\|T\|\|F\|_{p, \alpha}=O(1-|\xi|)^{(\alpha+2) / p-m-1},
$$

so that $g^{(m-2)} \in \Lambda_{\gamma}$ where $\gamma=(\gamma+2) / p-m$, by Theorem A and $g \in A(\Delta)$.

If $p=(\alpha+2) /(m+1)$, let $F(z)=d^{m+1} / d \xi^{m+1}(\xi /(1-\xi z))$. By a similar argument one can show that

$$
\left|g^{(m)}(\xi)\right|=O\left((1-|\xi|)^{-1}\right)
$$

and $g^{(m-2)} \in \Lambda_{*}$ by Theorem $B$ and $g \in A(\triangle)$.

To prove the converse we shall first show that if $g(z)=\sum_{k=0}^{\infty} b_{k} z^{k} \in \Lambda_{\gamma}$ where $\gamma=(\alpha+2) / p-m$, then for $f(z)=\sum_{n=0}^{\infty} a_{n} z^{n} \in A^{p, \alpha}, T(f)$ as defined in (3.1) exists. 
If $(\alpha+2) /(m+1)<p<(\alpha+2) / m$, let $\psi\left(\rho^{2}\right)=\sum_{k=0}^{\infty} a_{k}\left(b_{k} /(2 k+2)\right) \rho^{2 k}$. It is to be shown that $\psi\left(\rho^{2}\right)$ has a limit as $\rho \rightarrow 1$. We shall prove the existence of the limit by showing that

$$
\int_{0}^{1}\left|\left(\psi\left(\rho^{2}\right) \rho^{2}\right)^{\prime}\right| d \rho<\infty
$$

Set $h(z)=z^{m-2} g(z)$; then

$$
\begin{aligned}
\frac{1}{\pi} \int_{0}^{2 \pi} \int_{0}^{\rho} \bar{z} f_{[m-2]}\left(r e^{i \theta}\right) h^{m-1}\left(r e^{-i \theta}\right) d r d \theta & =\sum_{k=1}^{\infty} a_{k} b_{k} \rho^{2 k+1} \\
& =\left(\sum_{k=0}^{\infty} \frac{a_{k} b_{k} \rho^{2 k+2}}{2 k+2}\right)^{\prime}+a_{0} b_{0} \rho
\end{aligned}
$$

so

$$
\left(\psi\left(\rho^{2}\right) \rho^{2}\right)^{\prime}=\int_{0}^{2 \pi} \int_{0}^{\rho} e^{-i \theta} f_{[m-2]}\left(r e^{i \theta}\right) h^{(m-1)}\left(r e^{-i \theta}\right) d r d \theta-a_{0} b_{0} \rho .
$$

Using the assumption that $g^{(m-2)} \in \Lambda_{\gamma}$ gives

$$
\left|h^{(m-1)}\left(r e^{i \theta}\right)\right| \leqslant \frac{C}{(1-r)^{1-(\alpha+2) / p+m}}
$$

by Theorem A, consequently (3.5) gives

$$
\left|\left(\psi\left(\rho^{2}\right) \rho^{2}\right)^{\prime}\right| \leqslant C \int_{0}^{\rho}(1-r)^{(\alpha+2) / p-m-1} \int_{0}^{2 \pi}\left|f_{[m-2]}\left(r e^{i \theta}\right)\right| d \theta d r+\left|a_{0} b_{0}\right| .
$$

Hence by using Theorem F and D we have (3.4).

Finally, let $p=(\alpha+2) /(m+1)$ and $g^{(m-2)} \in \Lambda_{*} ;$ then (3.5) can be written in the form

$$
\left(\psi\left(\rho^{2}\right) \rho^{2}\right)^{\prime}=2 \int_{0}^{\rho} \int_{0}^{2 \pi} G\left(r e^{i \theta}\right) H\left(r e^{-i \theta}\right) d \theta d r-a_{0} b_{0} \rho
$$

where $H\left(r e^{i \theta}\right)=z h^{(m-1)}\left(r e^{i \theta}\right)$ and $G\left(r e^{i \theta}\right)=f_{[m-2]}\left(r e^{i \theta}\right)$. By Theorem F, $G \in$ $A^{2 / 3}$. Set $G(z)=\sum_{k=0}^{\infty} A_{k} z^{k}$; then by Theorem $\mathrm{F}$ again $G_{[1 / 2]} \in A^{4 / 5}$, so by Theorem $\mathrm{D}$

$$
\int_{0}^{1}(1-\rho)^{-(1 / 2)} J\left(\rho, G_{[1 / 2]}\right) d \rho<\infty
$$


Now, since $g^{(m-2)} \in \Lambda_{*}$, we have $\left|H^{[1 / 2]}\left(r e^{i \theta}\right)\right|=O(1 /(1-r))^{1 / 2}$, by Theorem B and Theorem G. Equation (3.6) can be written in the form

$$
\left(\psi\left(\rho^{2}\right) \rho^{2}\right)^{\prime}=2 \int_{0}^{\rho} \int_{0}^{2 \pi} G_{[1 / 2]}\left(r e^{i \theta}\right) H^{[1 / 2]}\left(r e^{i \theta}\right) d \theta d r-a_{0} b_{0} \rho
$$

consequently

$$
\left|\left(\psi\left(\rho^{2}\right) \rho^{2}\right)^{\prime}\right| \leqslant C \int_{0}^{\rho} \int_{0}^{2 \pi} \frac{1}{(1-r)^{1 / 2}}\left|G_{[1 / 2]}\left(r e^{i \theta}\right)\right| d \theta d r+\left|a_{0} b_{0}\right|
$$

hence

$$
\left(\psi\left(\rho^{2}\right) \rho^{2}\right)^{\prime} \leqslant C(1-\rho)^{-(1 / 2)} \int_{0}^{\rho} \int_{0}^{2 \pi}\left|G_{[1 / 2]}\left(r e^{i \theta}\right)\right| d \theta d r+\left|a_{0} b_{0}\right|
$$

and (3.7) gives (3.4).

To complete the proof, we need to show that if $g(z)=\sum_{k=0}^{\infty} b_{k} z^{k}$ is any analytic function for which $T(f)$ as defined in (3.1) exists for every $f(z)=\sum_{k=0}^{\infty} a_{k} z^{k} \in A^{p, \alpha}$, then $T \in\left(A^{p, \alpha}\right)^{*}$. For fixed $\rho \in[0,1)$, let $T(f)=\sum_{k=0}^{\infty}\left(a_{k} b_{k}\right) /(2 k+2) \rho^{2 k} ; T_{\rho}(f)$ is a linear functional on $A^{p, a}$. Also $T_{\rho}$ is bounded for each $\rho$ in $[0,1)$ by Theorem 1 . By hypothesis $\lim _{\rho \rightarrow 1} T_{\rho}(f)$ exists for each fixed $f \in A^{p, \alpha}$. Call this limit $T(f)$. By the uniform boundedness principle which holds for $A^{p, \alpha}[7, \mathrm{p} .45], \sup _{0 \leqslant \rho<1}\left\|T_{\rho}\right\|=C<\infty$. Thus $\left|T_{p}(f)\right| \leqslant C\|f\|_{p, \alpha}$ and by the continuity of $T_{p}$ in $[0,1],|T(f)| \leqslant C\|f\|_{p, \alpha}$. Therefore $f \in\left(A^{p, \alpha}\right)^{*}$.

\section{Equivalence of two Banach Spaces}

Let $\Lambda_{\alpha}^{n}(n=0,1, \ldots, 0<\alpha \leqslant 1)$ be the space of analytic functions $f(z)$ in $\triangle$ with $f, f^{1}, \ldots, f^{n} \in A(\Delta)$ and $f^{(n)} \in \Lambda_{\alpha}$ with the form

$$
\|f\|=\|f\|+\sup _{\substack{t, \theta \\ t>0}} \frac{\left|f^{(n)}\left(e^{i(\theta+t)}\right)-f^{(n)}\left(e^{i \theta}\right)\right|}{t^{\alpha}}
$$

$\Lambda_{\alpha}^{n}$ is a Banach space [2].

Let $\Lambda_{*}^{n}$ be the Banach space of functions analytic in $\Delta$ with $f, f^{*}, \ldots, f^{(n)} \in A$ and $f^{(n)} \in \Lambda_{*}$ normed by

$$
\|f\|=\|f\|_{\infty}+\sup _{\substack{t, \theta \\ t>0}} \frac{\left|f^{(n)}\left(e^{i(\theta+t)}\right)-2 f^{(n)}\left(e^{i \theta}\right)+f^{(n)}\left(e^{(\theta-t)}\right)\right|}{t} .
$$


Two Banach spaces $X$ and $Y$ are said to be equivalent if there is one-to-one linear mapping $L$ of $X$ onto $Y$ such that both $L$ and $L^{-1}$ are bounded. By the open mapping theorem it is sufficient that $L$ is bounded.

We have the following theorem:

TheOREM 2. If $(\alpha+2) /(m+1)<p<(\alpha+2) / m$, then the Banach space $\left(A^{p, \alpha}\right)^{*}$ and $\Lambda_{\gamma}^{m-2}$ with $\gamma=(\alpha+2) / p-m-1$ are equivalent. If $p=(\alpha+2) /(m+1)$, then $\left(A^{p, \alpha}\right)^{*}$ is equivalent to $\Lambda_{*}^{m-2}$.

Theorem 2 of [2] is a limiting case of Theorem 2 for $0<p<1 / 2$, and the question arises whether it holds for the case $1 / 2<p<1$.

Proof: Let $T \in\left(A^{p, \alpha}\right)^{*}$. By Theorem 1 the mapping $T \rightarrow g$ where $g$ is defined as in Theorem 1 , is a one-to-one linear mapping $L$ of $\left(A^{p, \alpha}\right)^{*}$ onto $\Lambda_{\gamma}^{m-2}\left(\Lambda_{*}^{m-2}\right)$. Then by (3.3)

$$
\left|g^{(m-1)}(\xi)\right| \leqslant C\|T\|(1-|\xi|)^{\beta-1}
$$

where $\beta=((\alpha+2) / p)-m$. Hence the proof of Theorem $5.1[3, p .74]$ shows that

$$
\left|g^{(m-2)}\left(e^{i(\theta+t)}\right)-g^{(m-2)}\left(e^{i \theta}\right)\right| \leqslant C\left(1+\frac{2}{\beta}\right)\|T\||t|^{\beta}
$$

We have $\|g\|_{\infty}=O(1)\|T\|$ by $(3.2)$, so

$$
\|g\| \leqslant C\|T\|
$$

Thus $g \in \Lambda_{\gamma}^{m-2}$ and $L(T)=g$, so

$$
\|L\|=\sup _{\|T\|=1} \frac{|L(T)|}{\|T\|}=\sup \frac{\|g\|}{\|T\|} \leqslant C .
$$

Thus $L$ is a bounded linear functional from $\left(A^{p, \alpha}\right)^{*}$ into $\Lambda_{\gamma}^{m-2}$ and Theorem 2 is proved.

\section{REFERENCES}

[1] K.K. Burchaev and V.G. Ryabykh, 'General form of linear functionals in $H_{p}^{1}$ spaces $0<p<1$ ', Siberian Math. J. 16 (1975), 678.

[2] P.L. Duren, B. Romberg and A.L. Shields, 'Linear functionals on $H^{p}$ spaces with $0<p<1$ ', J. Reine Angew Math. 238 (1969), 32-60.

[3] Peter L. Duren, Theary of $H^{p}$ Spaces (Academic Press, 1970).

[4] G.H. Hardy and J.E. Littlewood, 'Some properties of fractional integrals, II', Math. Z 34 (1932), 3-37. 
[5] C. Horowitz, 'Zero of functions in the Bergman spaces', Duke Math. J. 41 (1974), 693-710.

[6] A. Nakamura, F. Ohya and H. Watanabe, 'On some properties of functions in weighted Bergman spaces', Proc. Fac. Sci. Tokai Univ. 15 (1979), 33-40.

[7] W. Rudin, Functional analysis (MacGraw-Hill, 1973).

[8] J.H. Shapiro, 'Mackey topologies, reproducing kernels and diagonal maps on the Hardy and Bergman spaces', Duke Math. J. 43 (1976), 187-202.

[9] A.L. Shields and D.L. Williams, 'Bounded projections, duality and multipliers in spaces of analytic functions', Trans. Amer. Math. Soc. 162 (1971), 287-302.

[10] S.V. Shvedenko, 'On the Taylor coefficients of functions from Bergman spaces in the polydisc', Soviet Math. Dokl. 32 (1985), 118-121.

30 Rooks Run

Plymouth MA 02360

United States of America 